\begin{tabular}{|c|c|}
\hline \multicolumn{2}{|c|}{ Statistica Sinica Preprint No: SS-2016-0353R2 } \\
\hline Title & $\begin{array}{l}\text { Sparse Estimation of Generalized Linear Models (GLM) } \\
\text { via Approximated Information Criteria }\end{array}$ \\
\hline Manuscript ID & SS-2016-0353.R2 \\
\hline URL & http://www.stat.sinica.edu.tw/statistica/ \\
\hline DOI & $10.5705 /$ ss. 202016.0353 \\
\hline Complete List of Authors & $\begin{array}{l}\text { Xiaogang Su } \\
\text { Juanjuan Fan } \\
\text { Richard Levine } \\
\text { Martha Nunn and } \\
\text { Chih-Ling Tsai } \\
\end{array}$ \\
\hline Corresponding Author & Xiaogang Su \\
\hline E-mail & xiaogangsu@gmail.com, xsu@utep.edu \\
\hline
\end{tabular}


Statistica Sinica

\title{
Sparse Estimation of Generalized Linear Models (GLM) via Approximated Information Criteria
}

\author{
Xiaogang $\mathrm{Su}^{1}$, Juanjuan $\mathrm{Fan}^{2}$, Richard A. Levine ${ }^{2}$, \\ Martha E. Nunn ${ }^{3}$, and Chih-Ling Tsai ${ }^{4}$ \\ University of Texas at El Paso ${ }^{1}$, San Diego State University ${ }^{2}$ \\ Creighton University ${ }^{3}$, University of California at Davis ${ }^{4}$
}

\begin{abstract}
We propose a new sparse estimation method, termed MIC (Minimum approximated Information Criterion), for generalized linear models (GLM) in fixed dimensions. What is essentially involved in MIC is the approximation of the $\ell_{0}$-norm with a continuous unit dent function. Besides, a reparameterization step is devised to enforce sparsity in parameter estimates while maintaining the smoothness of the objective function. MIC yields superior performance in sparse estimation by optimizing the approximated information criterion without reducing the search space and is computationally advantageous since no selection of tuning parameters is required. Moreover, the reparameterization tactic leads to valid significance testing results that are free of post-selection inference. We explore the asymptotic properties of MIC and illustrate its usage with both simulated experiments and empirical examples.

Key words and phrases: BIC, generalized linear models, post-selection inference, sparse estimation, regularization, variable selection.
\end{abstract}

\section{Introduction}

Suppose that data $\mathcal{L}:=\left\{\left(y_{i}, \mathbf{x}_{i}\right): i=1, \ldots, n\right\}$ consist of $n$ i.i.d. copies of $\{y, \mathbf{x}\}$, where $y$ is the response variable and $\mathbf{x}=\left(x_{1}, \ldots, x_{p}\right)^{T} \in \mathbb{R}^{p}$ is the predictor vector. WLOG, we assume that the $x_{i j}$ 's are standardized throughout the paper. Consider the regression models that link the 
mean response $y$ and covariates $\mathbf{x}$ through its linear predictor $\mathbf{x}^{T} \boldsymbol{\beta}$ with $\boldsymbol{\beta}=\left(\beta_{1}, \ldots, \beta_{p}\right)^{T}$, e.g., generalized linear models (GLM; McCullagh and Nelder, 1989). Concerning variable selection, the true $\boldsymbol{\beta}$ can be sparse with some components being zeros. Sparse estimation is aimed to identify the zero components and estimate the nonzero ones in $\boldsymbol{\beta}$ at the same time. For simplicity, we assume that either there is no nuisance parameter involved or the nuisance parameters (e.g., scale or variance) and $\boldsymbol{\beta}$ are orthogonal (Cox and Reid, 1987). Hence we denote the log-likelihood function as $L(\boldsymbol{\beta})=\sum_{i=1}^{n} \log f\left(y_{i}, \mathbf{x}_{i} ; \boldsymbol{\beta}\right)$, where $f(y, \mathbf{x} ; \boldsymbol{\beta})$ denotes the probability density function of $(y, \mathbf{x})$.

Common variable selection methods can be formulated as an optimization problem

$$
\min _{\boldsymbol{\beta} \in \Omega}-2 \cdot L(\boldsymbol{\beta})+\lambda \sum_{j=1}^{p} \rho\left(\beta_{j}\right),
$$

where $\rho(\cdot) \geq 0$ denotes a penalty function applied to each individual component of $\boldsymbol{\beta} ; \lambda \geq 0$ is the penalty parameter; and $\Omega=\mathbb{R}^{p}$ is the search space or parameter space for $\boldsymbol{\beta}$. Different methods vary in the form of the penalty function and the way of determining the penalty parameter. In the classical best subset selection (BSS), the $\ell_{0}$ norm is used for the penalty, i.e., $\rho\left(\beta_{j}\right)=I\left(\beta_{j} \neq 0\right)$ or

$$
\sum_{j=1}^{p} \rho\left(\beta_{j}\right)=\sum_{j=1}^{p} I\left(\beta_{j} \neq 0\right)=\|\boldsymbol{\beta}\|_{0}=\operatorname{card}(\boldsymbol{\beta}),
$$

being the cardinality or the number of nonzero components in $\boldsymbol{\beta}$; the penalty parameter $\lambda_{0}$ is fixed as 2 in AIC (Akaike, 1974) or $\ln (n)$ in BIC (Schwarz, 1978). We shall focus more on the use of BIC for its superior empirical performance in variable selection widely reported in the literature. BSS essentially seeks the best model with minimum BIC. Owing to the discrete 
nature of the $\ell_{0}$ norm, the optimization is done in two steps: one first maximizes the log-likelihood $L(\boldsymbol{\beta})$ for each given sparsity structure or model ( $2^{p}$ in total) and then compare across all models. BSS is only feasible for small $p$, despite the availability of faster algorithms (Furnival and Wilson, 1974).

The second general approach to variable selection is regularization. One basic motivation of regularization is to change the discrete nature of BSS. For this purpose, different continuous penalty functions are proposed. In scenarios where the log-likelihood function is concave or can be converted so, the $\ell_{1}$ penalty $\|\boldsymbol{\beta}\|_{1}=\sum_{j=1}^{p}\left|\beta_{j}\right|$ in LASSO (Tibshirani, 1996) helps retain convexity of the optimization problem. LASSO requires strong assumptions in order to ensure selection consistency (Zhao and Yu, 2006) and induces bias in estimating the nonzero parameters. To make improvements, nonconvex penalties such as SCAD (Fan and Li, 2001) and MCP (Zhang, 2010) are proposed among others.

There are several difficulties with regularization. First of all, to induce sparsity in the estimated parameters, it is necessary for the penalty function $\rho(\beta)$ to have singularity at $\beta=0$. This makes the optimization in (1.1) non-smooth. As a result, many well-developed smooth optimization routines can not be used and one has to seek new algorithms. Although this issue has been successfully solved in the subsequent literature and efficient algorithms such as the homotopy (Osborne, Presnell, and Turlach, $2000)$ or LARS method (Efron et al., 2004) and the coordinate descent $(\mathrm{Fu}$, 1998. Friedman, Hastie, and Tibshirani, 2010, and Breheny and Huang, 2011) have become standard, it is of both methodological and practical interest to see if sparse estimation can be possibly formulated into a smooth optimization problem. Secondly, the penalty in regularization no longer 
corresponds well to model complexity represented by $\|\boldsymbol{\beta}\|_{0}$. Hence there is no simple rule as in $\mathrm{BIC}$ for determining the penalty parameter $\lambda$ and its choice has to be tuned. This leads to the two-step procedure in the practice of regularization: first compute the regularization path $\{\widehat{\boldsymbol{\beta}}(\lambda): \lambda \geq 0\}$ and then select the best tuning parameter $\lambda^{\star}$ by referring back to a criterion such as BIC (see, e.g., Wang, Li, and Tsai, 2007). In other words, regularization seeks minimum BIC from a much reduced search space, noting the fact that the regularization path is nothing but a one-dimensional curve in $\Omega$. Selecting $\lambda^{\star}$ not only consumes additional computational time, but also causes another statistically awkward issue concerning its inference. Although the best tuning parameter $\hat{\lambda}$ is data-dependent and hence clearly a statistic, no statistical inference is routinely done on $\lambda$, at least in the frequentist's approach.

Besides the above-mentioned deficiencies, another inherent problem with both BSS and regularization is the post-selection inference. Conventional statistical inference is made on the final model with selected variables or nonzero coefficients by ignoring the effect of model selection, which can be problematic as pointed out by Leeb and Pötscher (2005) among others. One knotty obstacle is that no statistical inference is available for parameters associated with those unselected variables in BSS or zero estimates in regularization. How to make valid post-selection inference is currently under intensive statistical research. See, e.g., Berk et al. (2013), Efron (2014), and Lockhart et al. (2014).

In this article, we study a new sparse estimation method for GLM, termed Minimum approximated Information Criterion (MIC), which was first proposed by $\mathrm{Su}(2015)$ in linear regression. The exposition in $\mathrm{Su}(2015)$ focuses on variable selection only; we shall expand the use of MIC in sparse 
estimation and the associated inference. The main idea of MIC is to introduce unit dent functions to approximate the $\ell_{0}$ norm in $(1.2)$. This leads to a smoothed version of BIC that can be directly optimized. A reparameterization step is then devised to enforce sparsity in parameter estimates while maintaining smoothness of the objective function. The formulation results in a non-convex yet smooth programming problem so that many readily available smooth optimization algorithms can be conveniently used to solve MIC. At the same time, the smoothness of the estimating equation allows us to derive valid significance testings on parameters that circumvents post-selection inference.

Compared to many currently available methods, MIC offers three major advantages in sparse estimation. First, it imitates BSS but extends its capacity to large $p$. Since MIC seeks optimization of BIC, albeit approximated, without reducing the search space to the regularization path, it offers superior performance, in the sense of minimum BIC, to many regularization methods in fixed dimensions. Secondly, MIC is computationally advantageous by avoiding selection of the tuning parameters. Thirdly, MIC facilitates statistical inference for both zero and non-zero coefficient estimates via the reparameterization trick.

We emphasize again that all our discussions are restricted to fixed dimensions. The remainder of this article is organized in the following manner. Section 2 presents the MIC method in detail. In Section 3, we explore its asymptotic properties under regular conditions. Section 4 presents simulation studies and data analysis examples. Section 5 concludes with a brief discussion.

\section{Minimizing the Approximated BIC}


MIC first approximates cardinality in the information criteria with a smooth unit dent function and then enforces sparsity with reparameterization. In its final form, MIC simply solves the following unconstrained smooth optimization problem:

$$
\min _{\boldsymbol{\gamma}}-2 L(\boldsymbol{\beta})+\log (n) \cdot \operatorname{tr}(\mathbf{W})
$$

where $\boldsymbol{\beta}=\mathbf{W} \boldsymbol{\gamma}$ and $\mathbf{W}=\operatorname{diag}\left(w_{j}\right)$ with $w_{j}=w\left(\gamma_{j}\right)=\tanh \left(a \gamma_{j}^{2}\right)$ for $j=1, \ldots, p$. The formulation of 2.1 involves a nonnegative parameters $a$, which controls the sharpness of approximation. Although asymptotic results suggest $a=O(n)$, the empirical performance of MIC is rather stable with respect to the choice of $a$ and hence $a$ is fixed $a$ priori. We shall explain the detailed procedure step-by-step in the ensuing subsections.

\subsection{Unit Dent Functions}

In a similar spirit to regularization, we desire to make the discrete BSS process continuous. While most regularization methods are based on optimization considerations, e.g., via convex relaxation of the $\ell_{0}$ norm to the $\ell_{1}$ norm in LASSO, MIC is mainly motivated by the idea of approximation. Specifically, we seek an approximation to the cardinality in 1.2 with a continuous or smooth surrogate function $w(\cdot)$.

For the convenience of presentation, we shall use $\beta$ as a generic notation for $\beta_{j}$ from time to time. The cardinality of $\boldsymbol{\beta}$ is $\sum I\left\{\beta_{j} \neq 0\right\}$ and hence it reduces to approximating the indicator function $I\{\beta \neq 0\}$. To this end, a suitable surrogate function $w(\beta)$ must be a unit dent function, as defined below.

Definition 1. Denote $\overline{\mathbb{R}}=R \cup\{-\infty, \infty\}$. A unit dent function is a continuous function $w: \overline{\mathbb{R}} \rightarrow[0,1]$ that satisfies the following properties: 
(i) $w(\cdot)$ is an even function such that $w(\beta)=w(-\beta)$;

(ii) $w(0)=0$ and $\lim _{\beta \rightarrow \infty} w(\beta)=1$;

(iii) $w(\beta)$ is increasing on $\mathbb{R}_{+}$.

The definition implies that $w(\beta)$ is decreasing on $\mathbb{R}_{-}$and $\lim _{\beta \rightarrow-\infty} w(\beta)=$ 1. If $w(\beta)$ is differentiable, then $\dot{w}(\beta) \geq 0$ on $\mathbb{R}+$ and $\dot{w}(\beta) \leq 0$ on $\mathbb{R}_{-}$. The $[0,1]$ range requirement essentially makes $w(\cdot)$ non-convex, but this is necessary in order for $\sum w(\beta)$ to approximate cardinality, namely, $\|$ $\boldsymbol{\beta} \|_{0} \approx \sum_{j=1}^{p} w\left(\beta_{j}\right)$. In addition, the condition $\lim _{|\beta| \rightarrow \infty} w(\beta)=1$ implies that $w(\beta)$ is approximately a constant function and hence $\dot{w}(\beta)=0$ for large $\beta$. As a consequence, when used as a penalty function, $w(\beta)$ essentially does not alter the related normal equations or score equations. Motivated by bump functions, we name $w(\cdot)$ a 'dent' function. A special family of bump functions, called mollifiers, are known as smooth approximations to the identity (Friedrichs, 1944). If a mollifier $\phi(\cdot)$ is normalized to have the range $[0,1]$, then $1-\phi(\cdot)$ is a unit dent function.

Let $\mathcal{D}$ denote the family of all unit dent functions. It can be easily seen that $\mathcal{D}$ is closed under operations such as composition and product. In particular, it is closed under power transformation. Namely, if $w(\beta) \in \mathcal{D}$, then $w^{k}(\beta) \in \mathcal{D}$ for $k \in \mathbb{N}$. It is worth noting that unit dent functions have appeared in the regularization literature. These include the truncated $\ell_{r}$ penalty studied by Shen, Pan, and Zhu (2012). The penalty functions SCAD (Fan and Li, 2001) and MCP (Zhang, 2010) can also be modified into unit dent functions. See Figure 1 for graphical illustrations of a number of unit dent functions.

To enforce sparsity, it is necessary for the penalty function to be nonsmooth with a singularity at $\beta=0$, as indicated by Fan and Li (2001). 
However, the use of smooth unit dent functions is advocated in MIC since the smoothness property allows us to capitalize on well-developed theories and methods in both optimization and statistical inference. We shall achieve achieve sparsity in a different way.

While many smooth unit dent functions can be considered, the hyperbolic tangent function is used in MIC for its simple form:

$$
w(\beta)=\tanh \left(a \beta^{2}\right)=\frac{\exp \left(2 a \beta^{2}\right)-1}{\exp \left(2 a \beta^{2}\right)+1}=2 \operatorname{logistic}\left(2 a \beta^{2}\right)-1 .
$$

Its derivatives are easily available, with the first two given by $\dot{w}(\beta)=2 a \beta(1-$ $\left.w^{2}\right)$ and $\ddot{w}(\beta)=2 a\left(1-w^{2}\right)\left(1-4 a \beta^{2} w\right)$. In addition, the $\tanh (\cdot)$ function is associated with the logistic or expit function which is widely used in statistics. A plot of $w(\beta)$ versus $\beta$ for different $a$ values is provided in Figure 1(d). It can be seen that a larger $a$ yields a sharper approximation to the indicator function $I\{\beta \neq 0\}$.

With the surrogate function $w(\beta)=\tanh \left(a \beta^{2}\right)$, one seeks to solve

$$
\min _{\boldsymbol{\beta}}-2 \cdot L(\boldsymbol{\beta})+\lambda_{0} \cdot \sum_{j=1}^{p} w\left(\beta_{j}\right)
$$

Expanding $L(\boldsymbol{\beta})$ at the MLE $\widehat{\boldsymbol{\beta}}$ and then using the fact that $\nabla L(\widehat{\boldsymbol{\beta}})=\mathbf{0}$, we have

$$
L(\boldsymbol{\beta}) \approx L(\widehat{\boldsymbol{\beta}})+(\boldsymbol{\beta}-\widehat{\boldsymbol{\beta}})^{T}\left\{\nabla^{2} L(\widehat{\boldsymbol{\beta}}) / 2\right\}(\boldsymbol{\beta}-\widehat{\boldsymbol{\beta}}),
$$

where $\nabla L(\widehat{\boldsymbol{\beta}})$ and $\nabla^{2} L(\widehat{\boldsymbol{\beta}})$ are the gradient vector and Hessian matrix of $L(\boldsymbol{\beta})$ evaluated at $\widehat{\boldsymbol{\beta}}$, respectively. Thus, the penalized optimization form in 2.3 can be viewed as the Lagrangian that corresponds to a constrained optimization problem:

$$
\min _{\boldsymbol{\beta}}(\boldsymbol{\beta}-\widehat{\boldsymbol{\beta}})^{T}\left\{-\nabla^{2} L(\widehat{\boldsymbol{\beta}})\right\}(\boldsymbol{\beta}-\widehat{\boldsymbol{\beta}}) \text { subject to } \sum_{j=1}^{p} w\left(\beta_{j}\right) \leq t_{0},
$$


for some $t_{0} \geq 0$. Figure 2 (a) presents a graphical illustration of the optimization problem (2.4) in the two-dimensional case. The objective function in 2.4 is an ellipsoid centered at MLE $\widehat{\boldsymbol{\beta}}$. As shown in the contour plots of Figure 2(a), the feasible set for the constraint $w\left(\beta_{1}\right)+w\left(\beta_{2}\right) \leq t_{0}$ contains both sharpened diamonds for large $t_{0}$ and discs for small $t_{0}$, resembling the grouped LASSO penalty (Bakin, 1999) as pointed out by a referee. By the Taylor expansion, $w(\beta)=a \beta^{2}+O\left(\beta^{6}\right)$ for $\beta \rightarrow 0$. This implies that sparsity may not be enforced. We shall address this issue in the next section. Hereafter, $w(\beta)$ is referred to the hyperbolic tangent penalty, unless otherwise explicitly stated.

\subsection{Reparameterization}

To enforce sparsity, we consider a reparameterization procedure originally motivated from the nonnegative garrotte (NG) of Breiman (1995). NG can be viewed as a sign-constrained regularization that is based on the decomposition $\beta=\operatorname{sgn}(\beta)|\beta|$. Supposing that the sign of each $\beta_{j}$ can be correctly specified by the MLE $\widehat{\boldsymbol{\beta}}$, it remains to estimate $\left|\boldsymbol{\beta}_{j}\right|$. Reparameterizing $\boldsymbol{\beta}=\operatorname{diag}\{\operatorname{sgn}(\widehat{\boldsymbol{\beta}})\} \boldsymbol{\gamma}$ for some nonnegative vector $\boldsymbol{\gamma}$ such that $\gamma_{j}=\left|\beta_{j}\right|$ leads to the NG formulation

$$
\min _{\boldsymbol{\gamma}}-2 L(\boldsymbol{\beta}) \text { s.t. } \sum_{j=1}^{p} \gamma_{j} \leq t \text { and } \gamma_{j} \geq 0
$$

where $t$ is a tuning parameter. One fundamental problem with sign-constrained regularization is that if any sign is wrongly specified by the initial estimator $\widehat{\boldsymbol{\beta}}$, which occurs often with real data owing to multicollinearity or other complexities, then it is not possible to make correction.

Our immediate aim is to introduce singularity to the penalty function at 0 . For this purpose, we consider the decomposition $\beta=\beta I\{\beta \neq 0\}$. Set 
$\gamma=\beta$ and approximate $I\{\gamma \neq 0\}$ with $w(\gamma)$. Namely,

$$
\gamma=\gamma I\{\gamma \neq 0\} \approx \gamma w(\gamma)
$$

This motivates the reparameterization $\beta_{j}$ as $\beta_{j}=\gamma_{j} w\left(\gamma_{j}\right)$ for $j=1, \ldots, p$. In matrix form, $\boldsymbol{\beta}=\mathbf{W} \boldsymbol{\gamma}$, where matrix $\mathbf{W}$ is defined earlier in (2.1). As shown in Figure 2(c), $\beta$ is an strictly increasing function of $\gamma$ and $\beta=\gamma$ except for a small neighborhood of 0 , in which a shrinkage on $|\beta|$ is imposed.

To see how the reparameterization helps with enforcing sparsity, consider the resulting optimization problem:

$$
\min _{\boldsymbol{\beta}}-2 \cdot L(\boldsymbol{\beta})+\ln (n) \cdot \sum_{j=1}^{p} w\left(\gamma_{j}\right) .
$$

Compared to $(2.3)$, the only change is that the penalty function $w(\cdot)$ is now applied to the reparameterized $\gamma_{j}$ instead of $\beta_{j}$. It is worth noting that the penalty function $w\left(\gamma_{j}\right)$ in 2.5 is an implicit function of $\beta_{j}$. Figure $2(\mathrm{~d})$ plots $w(\gamma)$ as a penalty function of $\beta$ for different values of $a$, which now shows a similar pattern to the non-convex SCAD or MCP penalty with a cusp at $\beta=0$. It can be easily verified that $w(\gamma)$ remains a unit dent function of $\beta$ that well approximates $I(\beta \neq 0)$.

The singularity at 0 can be further confirmed by calculating the derivatives of $w(\gamma)$ at $\beta$. Applying the chain rule gives

$$
\frac{d w(\gamma)}{d \beta}=\frac{d w(\gamma)}{d \gamma} \cdot \frac{d \gamma}{d \beta}=\frac{d w(\gamma)}{d \gamma} \cdot\left(\frac{d \beta}{d \gamma}\right)^{-1}=\frac{\dot{w}}{w+\gamma \dot{w}}
$$

where we denote $w=w(\gamma)$ and $\dot{w}=\dot{w}(\gamma)=2 a \gamma\left(1-w^{2}\right)$, and it follows $d \beta / d \gamma=w+\gamma \dot{w}$. The first derivative in 2.6 is expressed in terms of $\gamma$ via implicit differentiation since the explicit formula of $\gamma$ in terms of $\beta$ is unavailable. The validity of (2.6), however, requires $d \beta / d \gamma \neq 0$, which 
holds everywhere except at $\beta=0$. Similar arguments can be used to derive the form of the higher-order derivatives. For example, the second-derivative is given by

$$
\frac{d^{2} w(\gamma)}{d \beta^{2}}=\frac{w \ddot{w}-2 \dot{w}^{2}}{(w+\gamma \dot{w})^{3}}
$$

with $\ddot{w}=\ddot{w}(\gamma)=2 a\left(1-w^{2}\right)\left(1-4 a \gamma^{2} w\right)$, which again does not exist at $\beta=0$. It can be verified that $w(\gamma)$ is a smooth function of $\beta$ except at $\beta=0$.

It is worth mentioning that the property that the reparameterization $\beta=\gamma w(\gamma)$ helps enforce singularity at 0 holds for any smooth function in $\mathcal{D}$. We have utilized the differentiation of the inverse function to achieve this. Accordingly, the derivatives of $w(\gamma)$ as a function of $\beta$ exist everywhere except when $\beta=0$. There should be other ways of introducing singularities for smooth functions.

Figure 2(b) provides a two-dimensional illustration of the constrained optimization version that corresponds to $(2.5)$ :

$\min _{\boldsymbol{\beta}}(\boldsymbol{\beta}-\widehat{\boldsymbol{\beta}})^{T}\left\{-\nabla^{2} L(\widehat{\boldsymbol{\beta}})\right\}(\boldsymbol{\beta}-\widehat{\boldsymbol{\beta}})$ subject to $\operatorname{tr}(\mathbf{W}) \leq t_{0}$ with $\boldsymbol{\beta}=\mathbf{W} \boldsymbol{\gamma}$

The contour lines of the constraint $w\left(\gamma_{1}\right)+w\left(\gamma_{2}\right) \leq t$ (as a function of $\beta_{1}$ and $\beta_{2}$ now) become sharpened diamonds, which serves better for the variable selection purpose.

Besides achieving sparsity, the smooth formulation facilitated by reparameterization allows us to further capitalize on available results in both optimization theory and statistical inference and leads to some important conveniences and advantages. For the computation purpose, we shall estimate $\gamma$ instead by solving (2.1). Compared to 2.5 where the objective function is nonsmooth in $\boldsymbol{\beta}$, we have now switched the decision vector to 
$\boldsymbol{\gamma}$ instead of $\boldsymbol{\beta}$. Solving (2.1) is a smooth optimization problem and many standard algorithms can be applied. Estimation of $\gamma$ is meaningful in its own right. The fact that the correspondence between $\beta$ and $\gamma$ is one-toone with $\beta_{j}=0$ iff $\gamma_{j}=0$ allows us to derive significance testing results for $\boldsymbol{\beta}$ through $\boldsymbol{\gamma}$, which are free of post-selection inference. The objective function in (2.1) is smooth for estimating $\gamma$. Thus standard arguments in M-estimators can be applied for obtaining inference on $\gamma$. The detailed procedure will be explained in next section.

\section{Asymptotic Properties}

In this section, we first study the asymptotic oracle properties of the MIC estimator $\widetilde{\boldsymbol{\beta}}$, including its $\sqrt{n}$-consistency, selection consistency, and the asymptotic normality of its nonzero components. We then present significance testing on $\boldsymbol{\beta}$ via $\boldsymbol{\gamma}$, which is free of post-selection inference. Once again, we emphasize that all our discusses are restricted to the fixed $p$ scenarios.

\subsection{Oracle Properties of the MIC Estimator $\widetilde{\boldsymbol{\beta}}$}

For theoretical investigation, we consider the MIC estimator $\widetilde{\boldsymbol{\beta}}$ obtained from minimizing the objective function in 2.5

$$
Q_{n}(\boldsymbol{\beta})=-2 \frac{L(\boldsymbol{\beta})}{n}+\frac{\ln (n)}{n} \sum_{j=1}^{p} w\left(\gamma_{j}\right)
$$

where $L(\boldsymbol{\beta})=\sum_{i=1}^{n} l_{i}(\boldsymbol{\beta})$ with $l_{i}(\boldsymbol{\beta})=\log f\left(\mathbf{X}_{i}, Y_{i} ; \boldsymbol{\beta}\right)$. We shall denote $a$ as $a_{n}$ so that $\beta_{j}=\gamma_{j} w\left(\gamma_{j}\right)=\gamma_{j} \tanh \left(a_{n} \beta_{j}^{2}\right)$ and assume $a_{n}=O(n)$; this rate for $a_{n}$ will be manifested in the derivation.

Denote the true parameter as $\boldsymbol{\beta}_{0}=\left(\boldsymbol{\beta}_{0(1)}^{T}, \boldsymbol{\beta}_{0(0)}^{T}\right)^{T}$, where $\boldsymbol{\beta}_{0(1)} \in \mathbb{R}^{q}$ consists of all $q$ nonzero components and $\boldsymbol{\beta}_{0(0)}=\mathbf{0}$ consists of all the $(p-q)$ 
zero components. As generic notation, we use $\widetilde{\boldsymbol{\beta}}$ and $\widehat{\boldsymbol{\beta}}$ to denote the MIC and MLE estimators, respectively. Let $\mathbf{I}=\mathbf{I}\left(\boldsymbol{\beta}_{0}\right)$ be the expected Fisher information matrix for the whole model and let $\mathbf{I}_{1}$ be the Fisher information corresponding to the reduced true model setting $\boldsymbol{\beta}_{0(0)}=\mathbf{0}$. It is well known that $\mathbf{I}_{1}$ is the $q$-th principal submatrix of $\mathbf{I}$. The following theorem shows that, under regularity conditions, there exists a local minimizer $\widetilde{\boldsymbol{\beta}}$ of $Q_{n}(\boldsymbol{\beta})$ that is $\sqrt{n}$-consistent and this $\sqrt{n}$-consistent $\widetilde{\boldsymbol{\beta}}$ enjoys the 'oracle' property.

Theorem 1. Let $\left\{\left(\mathbf{X}_{i}, Y_{i}\right): i=1, \ldots, n\right\}$ be $n$ i.i.d. copies from a density $f\left(\mathbf{X}, Y ; \boldsymbol{\beta}_{0}\right)$. Under the regularity conditions $(A)-(C)$ in Fan and Li (2001), we have

(i). ( $\sqrt{n}$-Consistency) there exists a local minimizer $\widetilde{\boldsymbol{\beta}}$ of $Q_{n}(\boldsymbol{\beta})$ that is $\sqrt{n}$-consistent for $\boldsymbol{\beta}_{0}$ in the sense that $\left\|\widetilde{\boldsymbol{\beta}}-\boldsymbol{\beta}_{0}\right\|=O_{p}\left(n^{-1 / 2}\right)$.

(ii). (Sparsity and Asymptotic Normality) Partition $\widetilde{\boldsymbol{\beta}}$ in $($ i $)$ as $\left(\widetilde{\boldsymbol{\beta}}_{(1)}^{T}, \widetilde{\boldsymbol{\beta}}_{(0)}^{T}\right)^{T}$ in a similar manner to $\boldsymbol{\beta}_{0}$. With probability tending to 1 as $n \rightarrow \infty$, $\widetilde{\boldsymbol{\beta}}$ must satisfy that

$$
\widetilde{\boldsymbol{\beta}}_{(0)}=\mathbf{0}
$$

and

$$
\sqrt{n}\left(\widetilde{\boldsymbol{\beta}}_{(1)}-\boldsymbol{\beta}_{0(1)}\right) \rightarrow N\left(\mathbf{0}, \mathbf{I}_{1}^{-1}\right)
$$

The results in Theorem 1 are analogous to Theorems $1 \& 2$ in Fan and $\operatorname{Li}(2001)$. It establishes that $\widetilde{\boldsymbol{\beta}}_{(0)}$ is selection consistent and $\widetilde{\boldsymbol{\beta}}_{(1)}$ is a best asymptotic normal (BAN; see, e.g., Serfling, 1980) estimator of $\boldsymbol{\beta}_{0(1)}$. We defer its proof to the Appendix. The standard errors (SE) for nonzero components in $\widetilde{\boldsymbol{\beta}}$ can be conveniently computed by replacing $\mathbf{I}_{1}$ in Theorem 1 (ii) with the observed Fisher information matrix (Efron and Hinkley, 1978) and 
plugging in $\widetilde{\boldsymbol{\beta}}$. Since $\widetilde{\boldsymbol{\beta}}$ is essentially an M-estimator, alternative sandwich SE formulas (Stefanski and Boos, 2002) are available, for which we shall not pursue further. However, as post-selection inferences, all these SE formulas are only available for nonzero components in $\widetilde{\boldsymbol{\beta}}$ and hence caution should be exercised.

\subsection{Inference on $\beta$ via $\gamma$}

MIC avoids the two-step estimation process in the best subset selection and regularization by completing both variable selection and parameter estimation in one single optimization step. This brings about a unique opportunity to address the fundamental post-selection inference problem.

Inference on zero components in $\boldsymbol{\beta}$ is unavailable in MIC. This is because asymptotic normality of M-estimators often involves a condition that the expected objective function $E\left\{Q_{n}(\boldsymbol{\beta})\right\}$ admits a second-order Taylor expansion at $\boldsymbol{\beta}_{0}$ whereas sparsity requires singularity of the penalty function $w(\gamma)$ as a function of $\beta$ at $\beta=0$. However, the reparameterisation helps us to circumvent this non-smoothness issue. The transformation $\beta=\gamma w(\gamma)$ is a bijection and $\beta=0$ iff $\gamma=0$. Therefore, testing $H_{0}: \beta_{j}=0$ is equivalent to testing $H_{0}: \gamma_{j}=0$. As the objective function of $\gamma, Q_{n}(\gamma)$ in 3.1 is smooth in $\gamma$. Therefore, the statistical properties of $\widetilde{\gamma}$ are readily available following standard M-estimation arguments, as given in the theorem below.

Theorem 2. Let $\gamma_{0}$ be the reparameterized parameter vector associated with $\boldsymbol{\beta}_{0}$ such that $\beta_{0 j}=\gamma_{0 j} w\left(\gamma_{0 j}\right)$. It follows that

$$
\left\|\boldsymbol{\gamma}_{0}-\boldsymbol{\beta}_{0}\right\|_{2}=O\left\{\exp \left(-2 a_{n} \min _{1 \leq j \leq q} \gamma_{0 j}^{2}\right)\right\}
$$

Under the regularity conditions $(A)-(C)$ in Fan and Li (2001), we have

$$
\sqrt{n}\left[\mathbf{D}\left(\boldsymbol{\gamma}_{0}\right)\left(\widetilde{\gamma}-\boldsymbol{\gamma}_{0}\right)+\mathbf{b}_{n}\right] \stackrel{d}{\longrightarrow} N\left\{\mathbf{0}, \mathbf{I}^{-1}\left(\boldsymbol{\beta}_{0}\right)\right\} .
$$


where

$$
\mathbf{D}\left(\boldsymbol{\gamma}_{0}\right)=\left.\operatorname{diag}\left(w_{j}+\gamma_{j} \dot{w}_{j}\right)\right|_{\boldsymbol{\gamma}=\boldsymbol{\gamma}_{0}}=\operatorname{diag}\left(D_{j j}\right)
$$

and the asymptotic bias

$$
\mathbf{b}_{n}=\left\{-\nabla^{2} L\left(\boldsymbol{\beta}_{0}\right)\right\}^{-1} \frac{\ln (n)}{2}\left(\frac{\dot{w}_{j}}{w_{j}+\tilde{\gamma}_{j} \dot{w}_{j}}\right)_{j=1}^{p}=\left(b_{n j}\right)_{j=1}^{p}
$$

satisfy (i) $\lim _{n \rightarrow \infty} D_{j j}=I\left\{\beta_{0 j} \neq 0\right\}$ and (ii) $\mathbf{b}_{n}=o_{p}(1)$.

The proof of Theorem 2 is given in the Appendix. One practical implication of Theorem 2 is that both $\mathbf{D}\left(\boldsymbol{\gamma}_{0}\right)$ and $\mathbf{b}_{n}$ may be ignored in computing the standard errors of $\widetilde{\gamma}$. Furthermore, since $\left\|\widetilde{\gamma}-\boldsymbol{\beta}_{0}\right\| \leq\left\|\widetilde{\gamma}-\gamma_{0}\right\|+\|$ $\boldsymbol{\gamma}_{0}-\boldsymbol{\beta}_{0} \|=o_{p}(1), \widetilde{\boldsymbol{\gamma}}$ is a consistent estimator of $\boldsymbol{\beta}_{0}$ and can be used to replace $\boldsymbol{\beta}_{0}$ in estimating the Fisher information matrix. Thus, an asymptotic $(1-\alpha) \times 100 \%$ confidence interval for $\gamma_{0 j}$ can be simply given by

$$
\tilde{\gamma}_{j} \pm z_{1-\alpha / 2} \sqrt{\left(\mathbf{I}_{n}^{-1}(\widetilde{\gamma}) / n\right)_{j j}}
$$

where $\mathbf{I}_{n}$ denotes the observed Fisher information matrix and $z_{1-\alpha / 2}$ is the $(1-\alpha / 2)$-th percentile of $N(0,1)$. Significance testing on $\gamma_{0 j}$ can be done accordingly. There are alternative ways to derive the asymptotic variance of $\widetilde{\boldsymbol{\beta}}$. We numerically experimented a couple of other sandwich estimators and found that the simple formula in 3.5 performs very well empirically.

\section{Numerical Results}

In this section, we present simulation experiments and real data examples to illustrate MIC in comparison with other methods.

\subsection{Computational Issues}

MIC solves for $\widetilde{\gamma}$ by optimizing (2.1). Considering its nonconvex nature, a global optimization method is desirable. Mullen (2014) provides a comprehensive comparison of many global optimization algorithms currently 
available in $\mathrm{R}$ ( $\mathrm{R}$ Core Team, 2016). According to her recommendations, we have chosen the GenSA package (Xiang et al. 2013) that implements the generalized simulation annealing of Tsallis and Stariolo (1996), because of its superior performance in both identification of the true optimal point and computing speed. With estimated $\widetilde{\boldsymbol{\gamma}}$, the MIC estimator $\widetilde{\boldsymbol{\beta}}$ of $\boldsymbol{\beta}$ can be obtained immediately via the transformation $\widetilde{\boldsymbol{\beta}}=\widetilde{\mathbf{W}} \widetilde{\gamma}$, where $\widetilde{\mathbf{W}}=\operatorname{diag}\left(\tilde{w}_{j}\right)$ with $\tilde{w}_{j}=w\left(\tilde{\gamma}_{j}\right)$. Because of the shrinkage effect of the reparameterization around 0 , estimates $\tilde{\gamma}_{j}$ that are close to 0 would yield very small values of $\tilde{\beta}_{j}$, which can be virtually taken as 0 .

Implementation of MIC involves of the choice of $a_{n}$. In theory, the asymptotic results in Section 3.1 entail that $a_{n}=O(n)$. In order to apply the arguments of Fan and Li (2001), this $O(n)$ rate seems unique. See the proofs of Theorem 1 in the appendix. On the other hand, if one is willing to adjust the choice of $\lambda_{0}$, recall that the selection consistency of BIC holds for a wide range of $\lambda$ values, then the choice of $a_{n}$ can be more flexible. However, the conventional choice of $\lambda_{0}=\ln (n)$ is optimal in the Bayesian sense (Schwarz, 1978). Thus it is advisable to keep it as is. In practice, the empirical performance of MIC stays rather stable with respect to the choice of $a_{n}$, as demonstrated in $\mathrm{Su}(2015)$ for linear regression. The role of $a$ is quite different from the tuning parameter $\lambda$ in regularization that controls the penalty for complexity or the range of certain constraints. When $\lambda$ varies, the parameter estimates would change dramatically, which necessitates selection of $\lambda$. In MIC, $a_{n}$ is a shape or scale parameter in the unit dent function that modifies the sharpness of its approximation to the indicator function. The role of $a_{n}$ is largely similar to that of the parameter $a$ in SCAD (Fan and Li, 2001), where $a$ is fixed as $a=3.7$. In general, a larger $a_{n}$ value enforces a better approximation of the indicator 
function with the hyperbolic tangent function. On the other hand, a smaller $a_{n}$ is appealing for optimization purposes, by introducing more smoothness. Based on our numerical experiences, applying a $a$ value smaller than 1 leads to less stable and reduced performance. The performance of MIC stabilizes substantially when $a_{n}$ gets large, especially when it is 10 or above. On this basis, we recommend fixing $a$ to any value in $[10,50]$.

Four known methods are included for comparison with MIC: the best subset selection (BSS) with BIC, LASSO, SCAD, and MCP. The oracle estimate is also added as a benchmark. All the computations are done in $\mathrm{R}$ (R Development Core Team, 2015). Specifically, we have used the $\mathrm{R}$ package bestglm for BSS, lars and glmnet for LASSO, and ncvreg and SIS for SCAD and MCP. The default settings are used in these implementations, presuming that the default setting is the most recommendable.

\subsection{Simulated Experiments}

We generate data sets from the following three GLM models by using the same simulation settings as those of Zou and Li (2008). Specifically, the following three models are used:

Model A: $y \mid \mathbf{x} \sim N\{\mu(\mathbf{x}), 1\}$ with $\mu(\mathbf{x})=\mathbf{x}^{T} \boldsymbol{\beta}$,

Model B: $\quad y \mid \mathbf{x} \sim \operatorname{Bernoulli}\{\mu(\mathbf{x})\}$ with $\mu(\mathbf{x})=\operatorname{expit}\left(\mathbf{x}^{T} \boldsymbol{\beta}\right)$,

Model C: $y \mid \mathbf{x} \sim$ Poisson $\{\mu(\mathbf{x})\} \quad$ with $\mu(\mathbf{x})=\exp \left(\mathbf{x}^{T} \boldsymbol{\beta}\right)$,

where $\boldsymbol{\beta}=(3,1.5,0,0,2,0,0,0,0,0,0,0)^{T}$ in Models A and B, and $(1.2, .6,0$, $0, .8,0,0,0,0,0,0,0)^{T}$ in Model C. Each data set involves $p=12$ predictors that follow a multivariate normal distribution $N(\mathbf{0}, \boldsymbol{\Sigma})$ with $\boldsymbol{\Sigma}=\left(\sigma_{j j^{\prime}}\right)$ and $\sigma_{j j^{\prime}}=0.5^{\left|j-j^{\prime}\right|}$ for $j, j^{\prime}=1, \ldots, p$. In Model B, six binary predictors are created by setting $x_{2 j-1}:=I\left(x_{2 j-1}<0\right)$ for $j=1, \ldots, 6$. Thus, there are six continuous and six binary predictors in Model B. Each simulation includes 
two different sample sizes $n=100$ and $n=200$, and 500 realizations are generated from each model.

To apply the MIC method, we fix $\lambda_{0}=\ln (n)$ and $a_{n}=10$. Five performance measures are used for making comparisons. The first one is the empirical model error (ME), defined as $\mathrm{ME}=\sum_{i=1}^{n}\left(\mu_{i}-\hat{\mu}_{i}\right)^{2} / n$, where $\mu_{i}$ is given in (4.1) and $\hat{\mu}_{i}$ is obtained by plugging in the estimate of $\boldsymbol{\beta}$. We compute ME based on an independent test sample of size $n=500$ and then report the averaged ME over 500 realizations. The other measures are the average model size (Size; defined as the number of nonzero parameter estimates), the average number of false positives (FP; defined as the number of nonzero estimates for zero parameters), the average number of false negatives (FN; defined as the number of zero estimates for nonzero parameters), and the proportion of correct selections $(\mathrm{C})$.

Table 1 indicates that MIC performs similarly to BSS across all three models. In addition, all performance measures of MIC improve as the sample size increases. By comparing MIC against the other regularization methods, we find that MIC outperforms them in general, except for the Gaussian linear regression case where its performance is only comparable. We think this is mainly because the objective function of MIC involves the Gaussian profile likelihood $n \ln \|\mathbf{y}-\mathbf{X} \boldsymbol{\beta}\|^{2}$, which is nonconvex, while regularization methods can work with the convex least squares problem $\|\mathbf{y}-\mathbf{X} \boldsymbol{\beta}\|^{2}$ directly. Nevertheless, they all have to deal with the same log-likelihood function in Model B and C. Note that no implementation of MCP is available for the log-linear regression, hence it is not presented for Model C. In sum, MIC not only enjoys computational efficiency, but also demonstrates excellent finite sample performance.

We next evaluates the standard error formula for nonzero parameter 
estimates. Table 2 presents the median absolute deviation (MAD) value of $\widetilde{\boldsymbol{\beta}}_{(1)}$ out of 500 runs, which provides a more robust estimates of its standard deviation. This MAD value matches reasonably well with the median of standard errors of $\widetilde{\boldsymbol{\beta}}_{(1)}$. Also presented is the MAD of standard errors. Table 3 presents the empirical size and power results in testing $H_{0}: \gamma_{j}=0$ at the significance level $\alpha=0.05$, together with the coverage of $95 \%$ confidence intervals, over 1,000 simulation runs. It can be seen that the proposed testing procedure has empirical sizes close to the nominal level 0.05 while showing exceptional empirical powers and reasonable coverage.

\subsection{Real Data Examples}

We consider the diabetes data (Efron et al., 2004), the heart data (Hastie, Tisshirani, and Friedman, 2009), and the fish count data (available from http://www.ats.ucla.edu/stat/data/fish.csv) to illustrate linear regression, logistic regression, and log-linear regression models, respectively.

Table 4 shows that MIC (with $\lambda_{0}=\ln (n)$ and $a=10$ ) provides the similar selection as the best subset selection across all three examples. In addition, the resulting MIC estimates and their standard errors are quite close to these of the BIC model. This finding indicates that MIC approximates the best subset selection method well. This, together with MIC's computational efficacy, allows us to employ MIC on data with large numbers of covariates, even when BSS becomes infeasible. In the diabetes data, it is particularly interesting to note that the sign of the parameter estimate on hdl is positive under the full model fitting, but becomes negative in MIC and several other methods. This sign change could be problematic for sign-constrained methods such as NG (Breiman, 1995), but it comes out 
naturally in MIC. Furthermore, MIC is computationally much advantageous by design. See Table 1 in the Supplementary Materials for a comparison study on computing time.

To illustrate the stability of MIC with respect to the value of $a$, we obtain the MIC estimates for $a \in\{1,5,10,15, \ldots, 100\}$ and then plot them in Figure 3. While there are some reasonable minor variations mainly owning to the non-convex optimization nature, almost all the estimated coefficients are quite steady in all three examples, showing that the MIC estimation is generally robust to the choice of $a$.

\section{Discussion}

MIC is the first method that does sparse estimation by explicitly approximating $\mathrm{BIC}$. BIC is optimal in two aspects: it approximates the posterior distribution of candidate models besides being selection-consistent. This is why BIC has been used as an ultimate yardstick in many variable selection and regularization methods. MIC extends the best subset selection to scenario with large $p$ by optimizing an approximated BIC. Formulated as a smooth optimization problem, MIC is computationally advantageous to the discrete-natured best subset selection and enjoys the additional benefit in avoiding the post-selection inference. Moreover, the search space in $\mathrm{MIC}$ remains to be the entire parameter space. This explains why we expect MIC to outperform many regularization methods that have a much reduced search space for minimum BIC. By borrowing the knowledge of the fixed penalty parameter for model complexity in BIC, MIC circumvents the tuning parameter selection problem and hence is also computationally advantageous to regularization methods.

Although the hyperbolic tangent function has been used to approximate 
the cardinality in MIC, it can be replaced by other unit dent functions. Since one focus of this paper is on the variable selection consistency, we have adopted BIC by taking $\lambda_{0}=\ln (n)$. In contrast, if the aim is on the model selection efficiency or predictive accuracy, then we can adopt AIC by setting $\lambda_{0}=2$. It can be shown that the resulting MIC is selection-efficient by applying similar techniques to those used in Zhang, Li, and Tsai (2010). In sum, we can obtain variants of MIC by changing its penalty function $w$ and penalty parameter $\lambda_{0}$ to meet practical needs.

To broaden the usefulness of MIC, we would like to discuss three future research avenues. First, generalize MIC by accommodating the grouped or structured sparsity (see, e.g., Huang, Breheny, and Ma, 2012). Secondly, extend MIC to other complex model or dependence structures, such as finite mixture models, longitudinal data, and structural equation modelings (SEM). Similar ideas may be applied to approximate the effective degrees of freedom as well. In these settings, MIC can be particularly useful because the log-likelihood function is not concave and having convex penalties does not help anything with the optimization problem. Thirdly, develop the MIC method for data with diverging $p \rightarrow \infty$ yet $p / n \rightarrow 0$ (Fan and Peng, 2004) or ultra-high dimensions with $p \gg n$ (Fan and Lv, 2008) by approximating the extended or generalized BIC as pioneered by Chen and Chen (2008).

\section{Supplementary Materials}

In the Supplementary Materials, we outline the proofs of Theorems $1 \&$ 2 and provide more details about an R package glmMIC that implements MIC, on which basis a comparison study on computing time is also included. 


\section{References}

Akaike, H. (1974). A new look at model identification. IEEE Transactions an Automatic Control, 19: $716-723$.

Bakin, S. (1999) Adaptive regression and model selection in data mining problems. PhD Thesis. Australian National University, Canberra.

Berk, R., Brown, L., Buja, A., Zhang, K., and Zhao, L. (2013). Valid post-selection inference. The Annals of Statistics, 41, 802-837.

Breheny, P. and Huang, J. (2011). Coordinate descent algorithms for nonconvex penalized regression, with applications to biological feature selection. Annals of Applied Statistics, 5: $232-253$.

Breiman, L. (1995). Better subset regression using the nonnegative garrote. Technometrics, 37: $373-384$.

Chen, J. and Chen, Z. (2008). Extended Bayesian information criterion for model selection with large model spaces. Biometrika, 95: 759-771.

Cox, D. R. and Reid, N. (1987). Parameter orthogonality and approximate conditional inference (with discussion). Journal of the Royal Statistical Society, Series B, 49: 1-18.

Efron, B. (2014). Estimation and accuracy after model selection. Journal of the American Statistical Association, 109: 991-1007.

Efron, B., Hastie, T., Johnstone, I., and Tibshirani, R. (2004). Least angle regression (with discussion). The Annals of Statistics, 32: 407-499.

Efron, B. and Hinkley, D. V. (1978). Assessing the accuracy of the maximum likelihood estimator: observed versus expected Fisher information. Biometrika, 65: 457-482.

Fan, J. and Li, R. (2001). Variable selection via nonconcave penalized likelihood and its oracle properties. Journal of the American Statistical Association, 96: 1348-1360.

Fan, J. and Lv, J. (2008). Sure independence screening for ultrahigh dimensional feature space. 
Journal of the Royal Statistical Society, Series B, 70: 849-911.

Fan, J. and Peng, H. (2004). Nonconcave penalized likelihood with a diverging number of parameters. The Annals of Statistics, 32: 928-961.

Friedman, J. H., Hastie, T., and Tibshirani, R. (2010). Regularization paths for generalized linear models via coordinate descent. Journal of Statistical Software, 33(1).

Friedrichs, K. O. (1944). The identity of weak and strong extensions of differential operators. Transactions of the American Mathematical Society, 55: 132-151.

Furnival, G. M. and Wilson, R. W. (1974). Regression by Leaps and Bounds. Technometrics, 16: $499-511$.

Fu, W. (1998). Penalized regressions: the Bridge versus the Lasso. Journal of Computational and Graphical Statistics, 7(3): 397-416.

Hastie, T., Tibshirani, R. and Friedman, J. (2009). The Elements of Statistical Learning - Data Mining, Inference, and Prediction, 2nd Edition. Springer, New York.

Hoerl, A. E. and Kennard, R. W. (1970). Ridge regression: Biased estimation for nonorthogonal problems. Technometrics, 42: 80-86.

Huang, J., Breheny, P., and Shuangge Ma, S. (2012). A selective review of group selection in high-dimensional models. Statistical Science, 4: 481-499.

Leeb, H. and Pötscher, B. M. (2005). Model selection and inference: facts and fiction. Econometric Theory, 21, 21-59.

Lockhart, R., Taylor, J., Tibshirani, R., and Tibshirani, R. (2014). A significance test for the LASSO. The Annals of Statistics, 42, 413-468.

Loh, P.-L. and Wainwright, M. J. (2001). Regularized M-estimators with nonconvexity: Statistical and algorithmic theory for local optima. Journal of Machine Learning Research, 16: $559-616$.

McCullagh, P. and Nelder, J. A. (1989). Generalized Linear Models, 2nd ed. Chapman and Hall, 
London.

Mullen, K. M. (2014). Continuous global optimization in R. Journal of Statistical Software, $60(6)$

Osborne, M., Presnell, B., and Turlach, B. (2000). On the lasso and its dual. Journal of Computational and Graphical Statistics, 9: 319-337.

R Core Team (2016). R: A language and environment for statistical computing. R Foundation for Statistical Computing, Vienna, Austria. URL https://www.R-project.org/.

Schwarz, G. (1978). Estimating the dimension of a model. The Annals of Statistics, 6: 461-464.

Serfling, R. J. (1980). Approximation Theorems of Mathematical Statistics. New York, NY: John Wiley \& Sons.

Shen, X., Pan, W., and Zhu, Y. (2012). Likelihood-based selection and sharp parameter estimation. Journal of American Statistical Association, 107: 223-232.

Stefanski, L. A. and Boos, D. D. (2002). The calculus of M-estimation. The American Statistician, 56: 29-38.

$\mathrm{Su}, \mathrm{X}$. (2015). Variable selection via subtle uprooting. Journal of Computational and Graphical Statistics, 24(4): 1092-1113.

Tibshirani, R. J. (1996). Regression shrinkage and selection via the LASSO. Journal of the Royal Statistical Society, Series B, 58: 267-288.

Tsallis, C. and Stariolo, D. A. (1996). Generalized simulated annealing. Physica A, 233: 395406.

Wald, A. (1949). Note on the consistency of the maximum likelihood estiamte. Annals of Mathematical Statistics, 20: 595-601.

Wang, H., Li, R., and Tsai, C.-L. (2007). Tuning parameter selectors for the smoothly clipped absolute deviation method. Biometrika, 94: 553-568.

Weigend, A. S., Rumelhart, D. E., and Huberman, B. A. (1991). Generalization by weight- 


\section{REFERENCES}

elimination with application to forecasting, in Advances in Neural Information Processing Systems 3 (Denver 1990), R. P. Lippmann, J. E. Moody, and D. S. Touretzky, Editors, 875-882. Morgan Kaufmann, San Mateo, CA.

Xiang, Y., Gubian, S., Suomela, B., and Hoeng, J. (2013). Generalized simulated annealing for global optimization: The GenSA package. The $R$ Journal, 5(1).

Zhang, C.-H. (2010). Nearly unbiased variable selection under minimax concave penalty. The Annals of Statistics, 38: 894-942.

Zhang, Y., Li, R., and Tsai, C.-L. (2010). Regularization parameter selections via generalized information criterion. Journal of the American Statistical Association, 105: 312-323.

Zou, H. and Li, Y. (2008). One-step sparse estimates in nonconcave penalized likelihood models. The Annals of Statistics, 36: 1509-1533.

Zhao, P. and Yu, B. (2006). On model selection consistency of LASSO. Journal of Machine Learning, 7: 2541-2563.

Department of Mathematical Sciences, University of Texas, El Paso, TX 79968, U.S.A.

E-mail: xsu@utep.edu

Department of Mathematics and Statistics, San Diego State University, CA 92182, U.S.A.

E-mail: jjfan@mail.sdsu.edu; ralevine@sciences.sdsu.edu

Department of Periodontology, Creighton University, Omaha, NE 68178, U.S.A.

E-mail: MarthaNunn@creighton.edu

Graduate School of Management, University of California, Davis, CA 95616, U.S.A.

E-mail: cltsai@ucdavis.edu 


\section{REFERENCES}
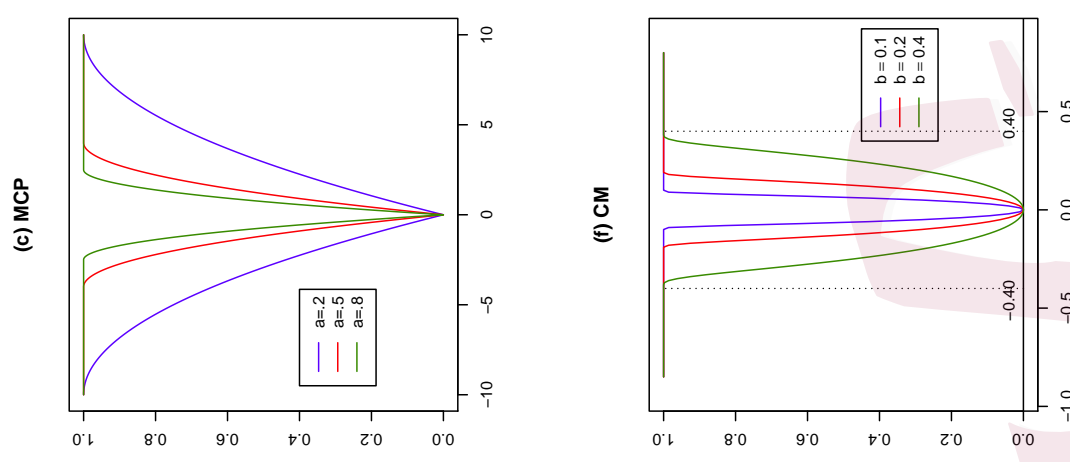

:

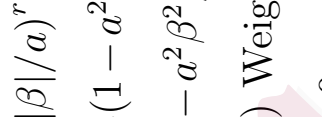

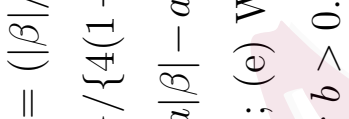

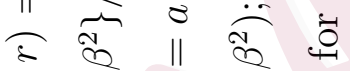

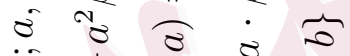

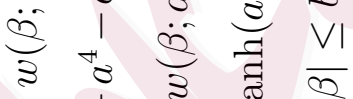
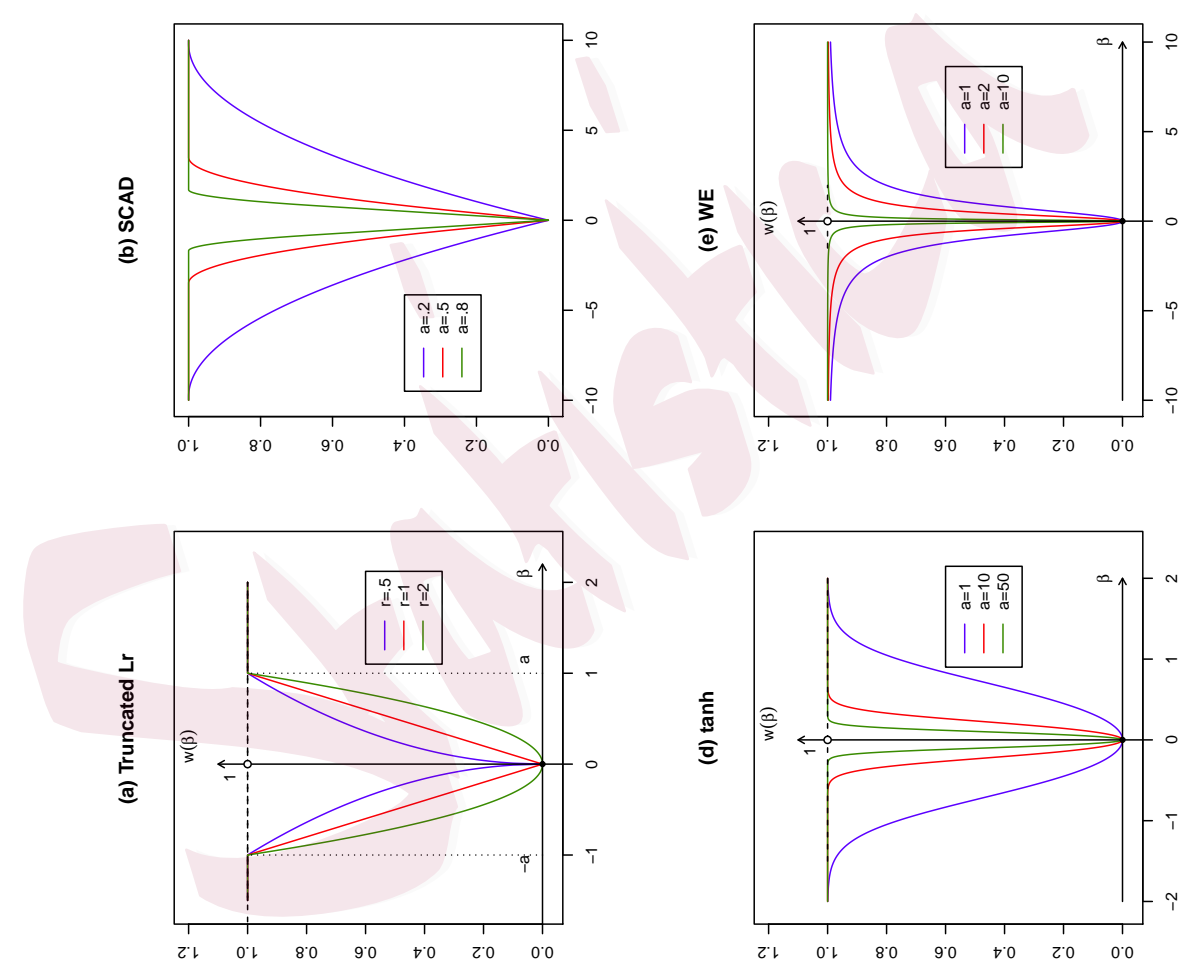

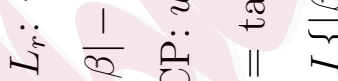

ఫ

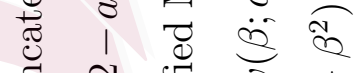

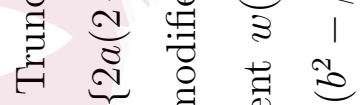

(ङ)

$\dot{0}$ VI $\stackrel{0}{0}$ I 1

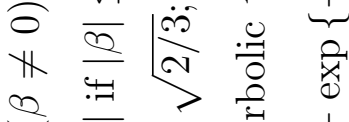

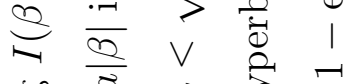

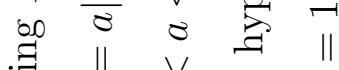

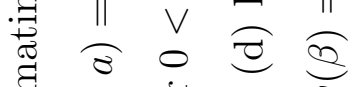

藏

范

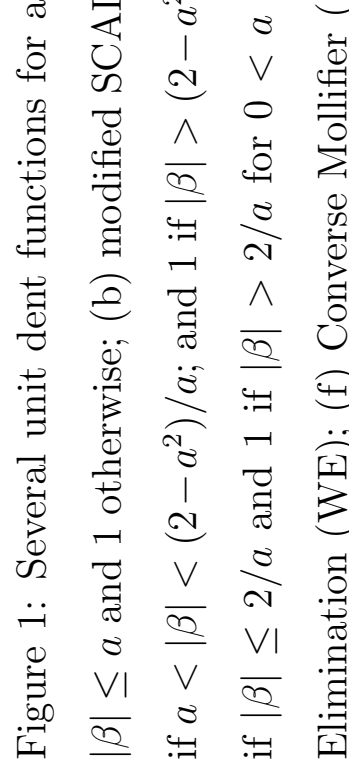


(a)

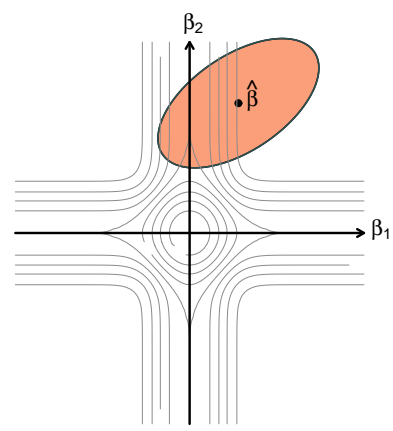

(c)

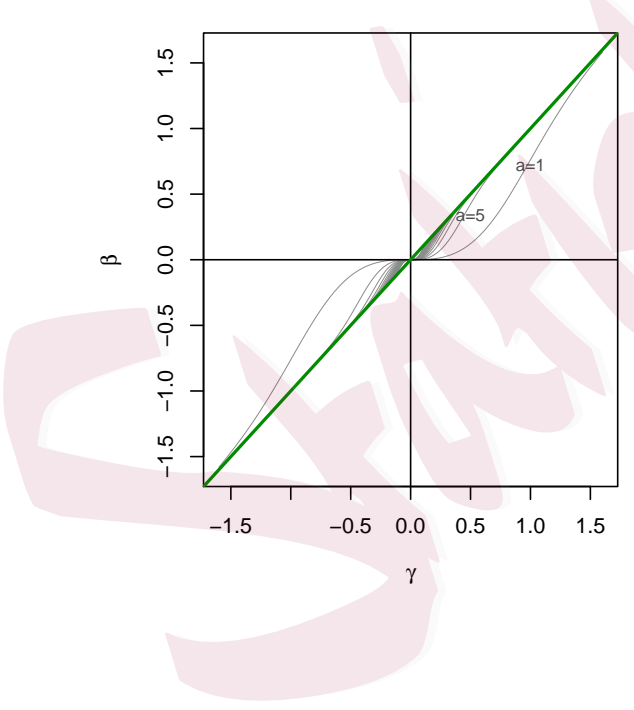

(b)

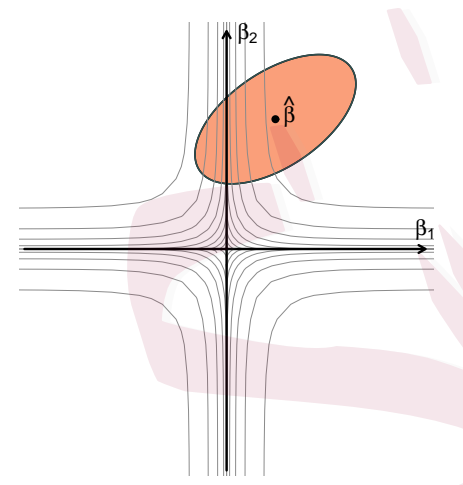

(d)

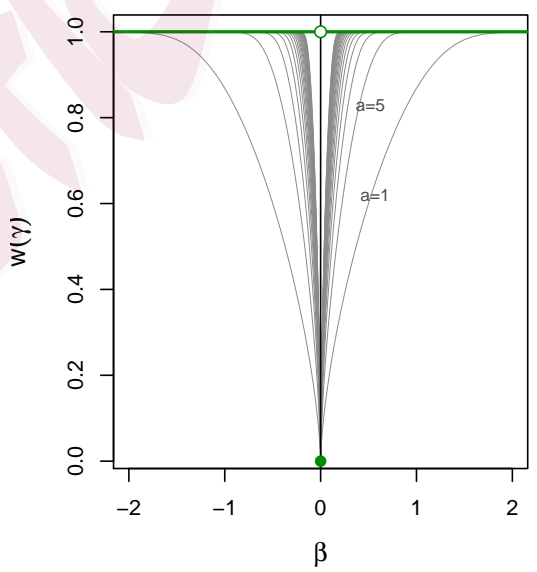

Figure 2: Illustration of the reparameterization step: (a) the contour plot for the optimization problem in the two-dimensional scenario before reparameterization; (b) the contour plot after reparameterization; (c) $\beta=\gamma w(\gamma)$ vs. $\gamma$; and $(\mathrm{d}) w(\gamma)$ as a penalty function for $\beta$. Different $a$ values in $\{1,5,10,15, \ldots, 100\}$ are used. 
(a) Linear Regression with Diabetes Data

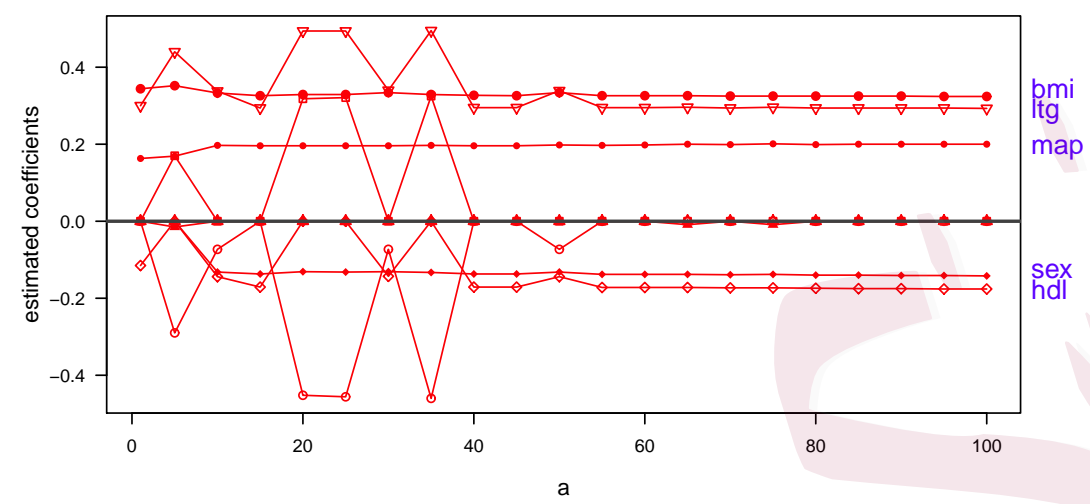

(b) Logistic Regression with Heart Data

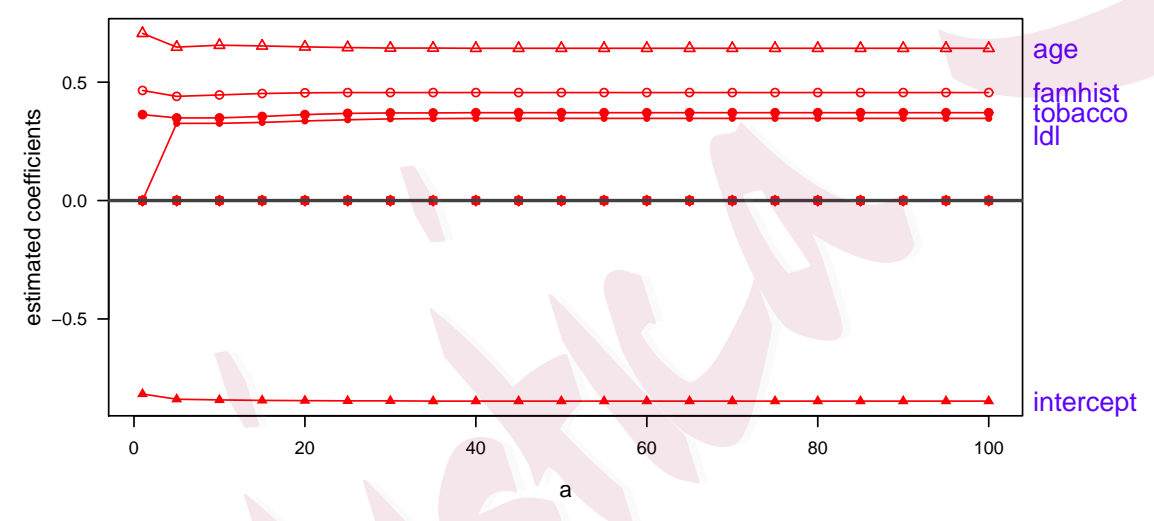

(c) Loglinear Regression with Fish Data

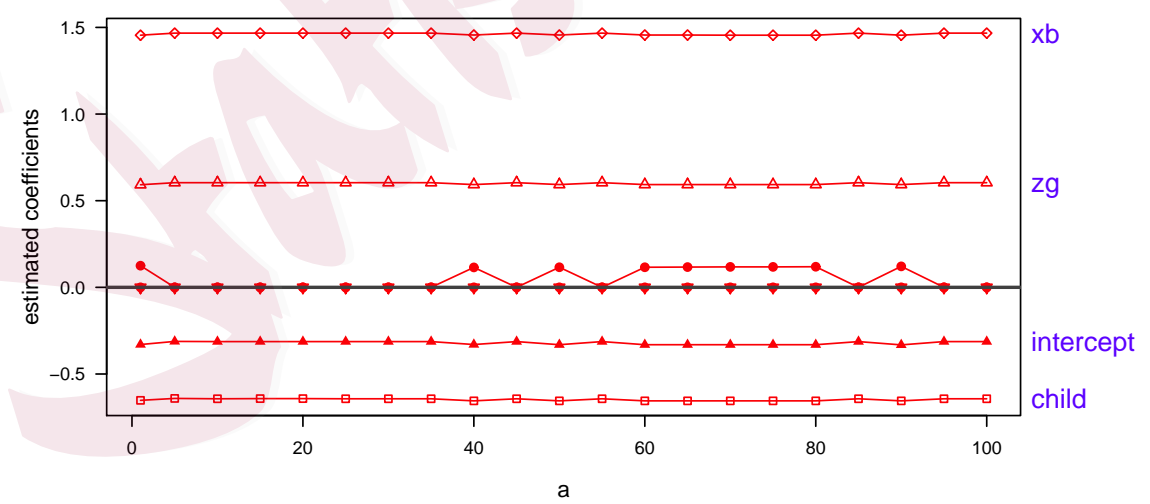

Figure 3: Illustrating the robustness of MIC with respect to the choice of $a$ in three real examples. The values of $a$ considered are $\{1,5,10,15, \ldots, 100\}$. 


\section{REFERENCES}

Table 1: Simulation results on MIC (with $\lambda_{0}=\ln (n)$ and $a=10$ ) in comparison with other methods. Reported quantities include the averaged model errors (ME), the averaged model size (Size), the average number of false positive variables (FP), the average number of false negative variables $(\mathrm{FN})$, the proportion of correct selections (C), all based on 500 realizations.

(a) Model A - Linear Regression

\begin{tabular}{|c|c|c|c|c|c|c|c|c|c|c|}
\hline \multirow[b]{2}{*}{ Method } & \multicolumn{5}{|c|}{$n=100$} & \multicolumn{5}{|c|}{$n=200$} \\
\hline & $\mathrm{ME}$ & Size & FP & $\mathrm{FN}$ & $\mathrm{C}$ & $\mathrm{ME}$ & Size & FP & $\mathrm{FN}$ & $\mathrm{C}$ \\
\hline MIC & 0.054 & 3.47 & 0.47 & 0.00 & 0.640 & 0.021 & 3.25 & 0.25 & 0.00 & 0.790 \\
\hline Oracle & 0.034 & 3.00 & 0.00 & 0.00 & 1.000 & 0.015 & 3.00 & 0.00 & 0.00 & 1.000 \\
\hline $\mathrm{BIC}$ & 0.055 & 3.35 & 0.35 & 0.00 & 0.710 & 0.022 & 3.19 & 0.19 & 0.00 & 0.834 \\
\hline LASSO & 0.085 & 6.09 & 3.09 & 0.00 & 0.092 & 0.039 & 6.23 & 3.23 & 0.00 & 0.102 \\
\hline SCAD & 0.045 & 3.58 & 0.58 & 0.00 & 0.752 & 0.022 & 3.71 & 0.71 & 0.00 & 0.752 \\
\hline MCP & 0.047 & 3.57 & 0.57 & 0.00 & 0.750 & 0.020 & 3.41 & 0.41 & 0.00 & 0.814 \\
\hline
\end{tabular}

(b) Model B - Logistic Regression

\begin{tabular}{|c|c|c|c|c|c|c|c|c|c|c|}
\hline \multirow[b]{2}{*}{ Method } & \multicolumn{5}{|c|}{$n=100$} & \multicolumn{5}{|c|}{$n=200$} \\
\hline & $\mathrm{ME}$ & Size & $\mathrm{FP}$ & $\mathrm{FN}$ & $\mathrm{C}$ & $\mathrm{ME}$ & Size & $\mathrm{FP}$ & $\mathrm{FN}$ & $\mathrm{C}$ \\
\hline $\mathrm{MIC}$ & 0.017 & 3.74 & 1.03 & 0.29 & 0.354 & 0.005 & 3.42 & 0.49 & 0.07 & 0.624 \\
\hline Oracle & 0.005 & 3.00 & 0.00 & 0.00 & 1.000 & 0.002 & 3.00 & 0.00 & 0.00 & 1.000 \\
\hline $\mathrm{BIC}$ & 0.015 & 3.40 & 0.67 & 0.27 & 0.514 & 0.005 & 3.21 & 0.28 & 0.06 & 0.766 \\
\hline LASSO & 0.023 & 6.54 & 3.79 & 0.25 & 0.012 & 0.012 & 7.32 & 4.37 & 0.05 & 0.018 \\
\hline SCAD & 0.019 & 3.69 & 1.09 & 0.41 & 0.206 & 0.012 & 3.92 & 1.11 & 0.19 & 0.278 \\
\hline MCP & 0.019 & 3.12 & 0.65 & 0.53 & 0.236 & 0.011 & 3.39 & 0.64 & 0.24 & 0.420 \\
\hline
\end{tabular}

(c) Model C - Log-Linear Regression

\begin{tabular}{|c|c|c|c|c|c|c|c|c|c|c|}
\hline \multirow[b]{2}{*}{ Method } & \multicolumn{5}{|c|}{$n=100$} & \multicolumn{5}{|c|}{$n=200$} \\
\hline & $\mathrm{ME}$ & Size & FP & $\mathrm{FN}$ & $\mathrm{C}$ & $\mathrm{ME}$ & Size & FP & $\mathrm{FN}$ & $\mathrm{C}$ \\
\hline MIC & 12.310 & 3.34 & 0.35 & 0.00 & 0.712 & 4.367 & 3.23 & 0.23 & 0.00 & 0.828 \\
\hline Oracle & 9.289 & 3.00 & 0.00 & 0.00 & 1.000 & 3.555 & 3.00 & 0.00 & 0.00 & 1.000 \\
\hline $\mathrm{BIC}$ & 25.884 & 3.39 & 0.39 & 0.00 & 0.714 & 4.897 & 3.23 & 0.23 & 0.00 & 0.826 \\
\hline LASSO & 600.821 & 1.55 & 0.37 & 1.81 & 0.184 & 348.182 & 1.46 & 0.18 & 1.72 & 0.282 \\
\hline SCAD & 40.753 & 4.08 & 1.08 & 0.00 & 0.336 & 12.843 & 3.64 & 0.64 & 0.00 & 0.528 \\
\hline MCP & 80.931 & 3.48 & 0.59 & 0.11 & 0.698 & 18.979 & 3.50 & 0.56 & 0.05 & 0.745 \\
\hline
\end{tabular}


Table 2: Simulation results on standard errors of nonzero $\widehat{\boldsymbol{\beta}}$ with $n=200$ over 500 simulation runs. Reported quantities are MAD of the parameter estimates, Median of the standard errors, and MAD of the standard errors.

(a) Model A - Gaussian Linear Regression

\begin{tabular}{cccccccc}
\hline & \multicolumn{3}{c}{ oracle } & & \multicolumn{3}{c}{ MIC } \\
\cline { 2 - 3 } \cline { 6 - 8 } \cline { 6 - 8 } & MAD & Median SE & MAD SE & & MAD & Median SE & MAD SE \\
\hline$\beta_{1}$ & 0.083 & 0.082 & 0.006 & & 0.083 & 0.082 & 0.006 \\
$\beta_{2}$ & 0.084 & 0.082 & 0.006 & & 0.087 & 0.082 & 0.006 \\
$\beta_{5}$ & 0.072 & 0.072 & 0.005 & & 0.073 & 0.072 & 0.005 \\
\hline
\end{tabular}

(b) Model B - Logistic Regression

\begin{tabular}{cccccccc}
\hline & \multicolumn{3}{c}{ oracle } & & \multicolumn{3}{c}{ MIC } \\
\cline { 2 - 3 } \cline { 6 - 8 } \cline { 6 - 8 } & MAD & Median SE & MAD SE & & MAD & Median SE & MAD SE \\
\hline$\beta_{1}$ & 0.528 & 0.475 & 0.086 & & 0.529 & 0.492 & 0.094 \\
$\beta_{2}$ & 0.399 & 0.389 & 0.048 & & 0.448 & 0.407 & 0.064 \\
$\beta_{5}$ & 0.380 & 0.356 & 0.059 & & 0.405 & 0.367 & 0.061 \\
\hline
\end{tabular}

(c) Model C - Loglinear Regression

\begin{tabular}{cccccccc}
\hline & \multicolumn{3}{c}{ oracle } & & \multicolumn{3}{c}{ MIC } \\
\cline { 2 - 3 } \cline { 6 - 8 } \cline { 6 - 8 } & MAD & Median SE & MAD SE & & MAD & Median SE & MAD SE \\
\hline$\beta_{1}$ & 0.037 & 0.036 & 0.007 & & 0.037 & 0.036 & 0.007 \\
$\beta_{2}$ & 0.039 & 0.039 & 0.007 & & 0.040 & 0.039 & 0.007 \\
$\beta_{5}$ & 0.032 & 0.032 & 0.006 & & 0.033 & 0.033 & 0.006 \\
\hline
\end{tabular}


REFERENCES

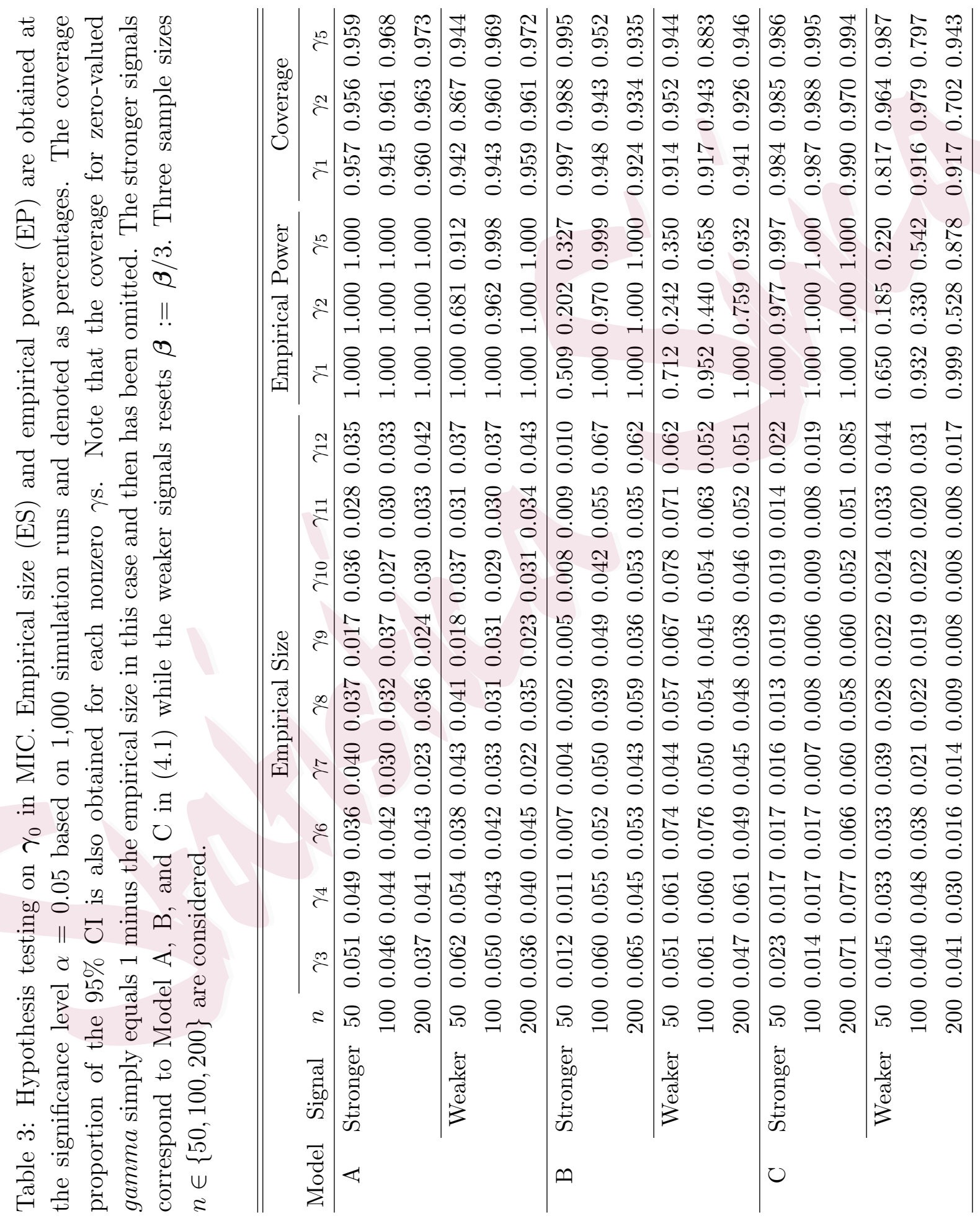




\section{REFERENCES}

Table 4: Illustration with real data examples. The p-values ${ }^{\star}$ in MIC are based on testing $H_{0}: \gamma_{0 j}=0$.

(a) Linear Regression with Diabetes Data

\begin{tabular}{|c|c|c|c|c|c|c|c|c|c|c|}
\hline & \multicolumn{2}{|c|}{ Full Model } & \multicolumn{2}{|c|}{ Best Subset } & \multicolumn{3}{|c|}{$\mathrm{MIC}$} & \multirow[b]{2}{*}{ LASSO } & \multirow[b]{2}{*}{ SCAD } & \multirow[b]{2}{*}{$\mathrm{MCP}$} \\
\hline & $\hat{\beta}_{j}$ & $\mathrm{SE}$ & $\hat{\beta}_{j}$ & $\mathrm{SE}$ & p-value ${ }^{\star}$ & $\hat{\beta}_{j}$ & $\mathrm{SE}$ & & & \\
\hline age & -0.006 & 0.037 & & & 1.000 & & & & & \\
\hline sex & -0.148 & 0.038 & -0.146 & 0.037 & 0.000 & -0.137 & 0.037 & -0.122 & -0.149 & -0.143 \\
\hline bmi & 0.321 & 0.041 & 0.323 & 0.040 & 0.000 & 0.325 & 0.040 & 0.323 & 0.321 & 0.328 \\
\hline map & 0.200 & 0.040 & 0.202 & 0.039 & 0.000 & 0.196 & 0.039 & 0.184 & 0.199 & 0.197 \\
\hline tc & -0.489 & 0.257 & & & 1.000 & & & -0.064 & -0.381 & \\
\hline ldl & 0.294 & 0.209 & & & 1.000 & & & & 0.216 & -0.067 \\
\hline hdl & 0.062 & 0.131 & -0.179 & 0.041 & 0.011 & -0.171 & 0.041 & -0.138 & & -0.179 \\
\hline tch & 0.109 & 0.100 & & & 1.000 & & & & 0.080 & \\
\hline ltg & 0.464 & 0.106 & 0.293 & 0.041 & 0.000 & 0.294 & 0.041 & 0.318 & 0.426 & 0.300 \\
\hline glu & 0.042 & 0.041 & & & 1.000 & & & 0.034 & 0.041 & 0.030 \\
\hline
\end{tabular}

(b) Logistic Regression with Heart Data

\begin{tabular}{|c|c|c|c|c|c|c|c|c|c|c|}
\hline & \multicolumn{2}{|c|}{ Full Model } & \multicolumn{2}{|c|}{ Best Subset } & \multicolumn{3}{|c|}{ MIC } & \multirow[b]{2}{*}{ LASSO } & \multirow[b]{2}{*}{ SCAD } & \multirow[b]{2}{*}{ MCP } \\
\hline & $\hat{\beta}_{j}$ & $\mathrm{SE}$ & $\hat{\beta}_{j}$ & $\mathrm{SE}$ & p-value ${ }^{\star}$ & $\hat{\beta}_{j}$ & SE & & & \\
\hline intercept & -0.845 & 0.120 & -0.847 & 0.120 & 0.000 & -0.842 & 0.119 & -0.787 & -0.846 & -0.844 \\
\hline sbp & 0.118 & 0.115 & & & 1.000 & & & 0.041 & & 0.062 \\
\hline tobacco & 0.365 & 0.120 & 0.371 & 0.117 & 0.001 & 0.349 & 0.116 & 0.299 & 0.371 & 0.369 \\
\hline ldl & 0.383 & 0.119 & 0.347 & 0.112 & 0.001 & 0.326 & 0.111 & 0.271 & 0.350 & 0.368 \\
\hline famhist & 0.463 & 0.111 & 0.456 & 0.110 & 0.000 & 0.446 & 0.110 & 0.371 & 0.456 & 0.460 \\
\hline obesity & -0.146 & 0.123 & & & 1.000 & & & -0.011 & -0.086 & \\
\hline alcohol & 0.015 & 0.109 & & & 1.000 & & & & & \\
\hline age & 0.621 & 0.149 & 0.643 & 0.142 & 0.000 & 0.656 & 0.142 & 0.544 & 0.645 & 0.632 \\
\hline
\end{tabular}

(c) Log-Linear Regression with Fish Data

\begin{tabular}{|c|c|c|c|c|c|c|c|c|c|c|}
\hline & \multicolumn{2}{|c|}{ Full Model } & \multicolumn{2}{|c|}{ Best Subset } & \multicolumn{3}{|c|}{ MIC } & \multirow[b]{2}{*}{ LASSO } & \multirow[b]{2}{*}{ SCAD } & \multirow[b]{2}{*}{$\mathrm{MCP}$} \\
\hline & $\hat{\beta}_{j}$ & $\mathrm{SE}$ & $\hat{\beta}_{j}$ & $\mathrm{SE}$ & p-value ${ }^{\star}$ & $\hat{\beta}_{j}$ & $\mathrm{SE}$ & & & \\
\hline intercept & -0.360 & 0.090 & -0.313 & 0.073 & 0.000 & -0.304 & 0.073 & 0.357 & -0.007 & \\
\hline nofish & -0.033 & 0.059 & & & 1.000 & & & & 0.027 & \\
\hline livebait & 0.129 & 0.090 & & & 1.000 & & & & & \\
\hline camper & -0.010 & 0.051 & & & 1.000 & & & & & \\
\hline persons & 0.047 & 0.057 & & & 1.000 & & & & & \\
\hline child & -0.653 & 0.103 & -0.643 & 0.098 & 0.000 & -0.638 & 0.098 & & -0.645 & -0.648 \\
\hline $\mathrm{xb}$ & 1.447 & 0.064 & 1.467 & 0.034 & 0.000 & 1.464 & 0.034 & 0.331 & 1.462 & 1.460 \\
\hline $\mathrm{zg}$ & 0.659 & 0.136 & 0.604 & 0.067 & 0.000 & 0.604 & 0.067 & & 0.601 & 0.598 \\
\hline$x b: z g$ & -0.034 & 0.059 & & & 1.000 & & & 0.176 & & \\
\hline
\end{tabular}

